\title{
An Approach to Explore the Role of Color Models and Color Descriptors in the Optimization of Semantic Gap in Content based Image Retrieval
}

\author{
Pranoti Mane \\ Associate Professor \\ M.E.S. College of Engineering, \\ Pune, India
}

\author{
Narendra Bawane \\ Principal \\ S.B.J.College of Engg.,Research \& Management, \\ Nagpur, India
}

\begin{abstract}
Content based image retrieval (CBIR) systems retrieve images based on their primitive features such as color, texture, shape etc. The semantic gap is defined as the inconsistency between the image retrieval based on these low level image features and high level human semantics. In this paper, the comparative analysis of various color model transformations is presented with the help of our proposed methods based on three color descriptors i.e. color histogram, color moments and color coherence vectors to determine the applicability of these models and descriptors for the reduction of semantic gap. Support vector machines are used to classify images into different semantic classes. The results are inferred with the help of performance parameters like precision, recall, and mean average precision. Experimental results suggest that the proposed approach gives a good evaluation of the applicability of color models as well as color descriptors for optimization of semantic gap in CBIR.
\end{abstract}

\section{General Terms}

Image Processing, Pattern Recognition, Classification

\section{Keywords}

Color models, Content based image retrieval (CBIR), Mean Average Precision, Semantic gap, Support vector machines.

\section{INTRODUCTION}

Content based image retrieval system(CBIR)[1-7] is stated as the retrieval of images based on low level or primitive image features such as shape, color, texture, etc. This retrieval is usually based on the similarity index rather than on the exact match [2]. The critical issue in CBIR system is the development of an effective and efficient feature extraction method for image representation that complies with the human perception subjectivity. This subjectivity occurs in all semantic levels while analyzing images because different users in same situation or the same user in different circumstances may analyze or classify the same image differently. This inconsistency between the image retrieval based on low level image features and high level human semantics is termed as the Semantic Gap [6-10]. Color is one of the most extensively used visual features in image retrieval. Color features are relatively strong to the viewing angle, translation and rotation of the regions of interest in an image. Although color is certainly not the most important primitive feature when it comes to semantic retrieval, but if spatial distribution of colors is considered while extracting color features for an image, it constitutes a good launching point for future work consisting of the development of more sophisticated image retrieval system which is directly supportive to optimize the semantic gap in content based image retrieval. In CBIR applications, color images can be treated in various ways by different color models [12-23]. A color model is a mathematical system to represent colors. No color model can be considered as universal because colors can be interpreted and represented in different ways. With a large variety of available color models, the foreseeable question that arises is how to select the color model and color descriptor that produce the best result for particular query image retrieval.

In this paper, the performance of a wide variety of color models and various color descriptors is evaluated. Overall 7 methods based on one or more color descriptors for color feature extraction are proposed. Every method is implemented for evaluating the performance of 10 color model transformations. Support vector machine classifiers [11,24] are used to classify the images into semantic classes like Manmade Indoor, Manmade Outdoor and Natural Outdoor. Initially the experiments are done on our own database comprising of 450 images from various categories like Store, bedroom, kitchen, snowy mountains, buses, coast, street, twilight, buildings and forest. Later the same experiments are done on a standard database of James Z. Wang et.al. [25] consisting of 1000 images of different categories like African people, buildings, beaches, buses, dinosaurs, elephants, roses, horses, snowy mountains and food plates. The results from this paper lead to novel methods to choose an appropriate color model with an appropriate combination of color descriptors for the optimization of semantic gap in CBIR.

The rest part of this paper is organized as follows. Section 2 focuses on the classification approach and related methodology for semantic gap reduction by reviewing various color models and color descriptors. Experimentation based on proposed methods is given in section 3 . In section 4 , the results are shown and the role of color models and color descriptors for the reduction of semantic gap in CBIR is discussed. Finally, the section 5 concludes the paper and throws light on the scope of the future work.

\section{CLASSIFICATION APPROACH AND RELATED METHODOLOGY FOR THE OPTIMIZATION OF SEMANTIC GAP}

Classification is one of the approaches to optimize the semantic gap in CBIR. Classification is the process of extracting low level image features (LLIFs) and mapping them to semantically meaningful concepts i.e. high level image features (HLIFs). LLIFs are not directly relevant to the user for image retrieval task whereas HLIFs have direct 
meaning to the user. LLIFs are to be extracted first in order to classify images into semantically meaningful classes. The extracted information should be unique for the particular image and similar to the information extracted from similar images. The primitive features required for image classification are color features and holistic structure features. If the spatial distribution is considered while extracting color features of an image, then color features can also be considered as holistic structure features [24]. Thus in this paper, the approach used for optimization of semantic gap in CBIR is based on feature extraction by color descriptors.

Color histogram $(\mathrm{CH})$, Color Moments $(\mathrm{CM})$ and Color coherence vectors (CCV) are used for feature extraction for CBIR. Color Histogram is an effective representation of the distribution of color contents in an image if the color pattern is unique as compared to rest of the data set . Computation of $\mathrm{CH}$ is easy and fast and it is robust to translation and rotation about the view axis. However it changes slowly with the scale, occlusion and viewing angle [27]. Color moments are efficient and effective in representing color distributions in the images and extensively used in many commercial retrieval systems e.g. QBIC [28, 29] especially when image contains just an object. Mathematically, the first three moments i.e. first order (mean), second order (variance) and third order (skewness) are stated as shown in equations (1) to (3).

$$
\mu_{i}=\frac{1}{N} \sum_{j=1}^{N} f_{i j}
$$

$$
\sigma_{i}=\sqrt{\frac{1}{N} \sum_{j=1}^{N}\left(f_{i j}-\mu_{i}\right)^{2}}
$$

$$
S_{i}=\sqrt[3]{\frac{1}{N} \sum_{j=1}^{N}\left(f_{i j}-\mu_{i}\right)^{3}}
$$

Where $f_{i j}$ is the value of the $i^{\text {th }}$ color component of image pixel $\mathrm{j}$ and $\mathrm{N}$ is the No. of pixels in the image. Color moments are a very compact representation compared to other color features. In some cases, an image may not be solely determined by its color histogram or the distribution of colors in it. One image may be consisting of a large No. of scattered pixels with a particular color while another image may includes the same amount of pixels with that color but its pixels are gathered into the same area. The two different images may have same color histogram but different spatial arrangement of colors. $\mathrm{CCV}$ includes the information about spatial location of colors in an image[30].

Here, the retrieval efficiency is evaluated by using these color descriptors individually and it is marked that the performance is improved further when these descriptors are combined with one another.

It is important to explore different color models that are used to transform the color information of an image into a new coordinate system so that the comparison of color information can be made easier and perceptually more correct. Different color models that are analyzed in this paper are RGB, CMYk, I1I2I3, HSV, YIQ, YUV, L*a*b*, XYZ, YCbCr and HMMD.

Primary color model RGB [21, 23] is a device dependent color model which is usually used for color display and is non-linear with visual perception. However this model does not exhibit perceptible benefits over gray scale models in terms of convergence accuracy [21]. The Euclidean distance between colors in RGB space does not corresponds to the way the humans perceive the distance between colors [26]. Thus this may lead to unsatisfactory image classification. CMYK is another device dependent color model which is used for passive devices that deposit colored pigments on paper, such as color inkjet printers and copiers. XYZ is derived from RGB and it consists of tristimulus values which give the chromaticity components. Intensity and chromaticity information are strongly mixed in each of the RGB channels. Non correlated color model I1I2I3 is introduced by Ohta [31] after a colorimetric analysis of 8 images and realizes an efficient minimization of the inter channel correlations for natural images.

Color models based on luminance and chrominance components are YUV, YIQ, YCbCr, HSV, etc. YUV and YIQ models are used for analog television transmission whereas $\mathrm{YCbCr}$ is used for digital television transmission. $\mathrm{Y}$ is related to luminance and $\mathrm{U}, \mathrm{V}, \mathrm{I}, \mathrm{Q}, \mathrm{Cb}$ and $\mathrm{Cr}$ are related to chrominance. HMMD (Hue-Max-Min-Diff) is a perceptually uniform color model $[7,32]$ defined in three dimensions i.e. sum and difference axes and Hue angle. HSV color model is the non-linear but reversible transformation from RGB and it corresponds closely to the way humans describe and interpret colors.

One of the perceptual color models, $\mathrm{L}^{*} \mathrm{a}^{*} \mathrm{~b}^{*}$ also known as CIELAB, is a device independent color model which is also designed to correspond well with human perception of colors [26]. Here, a color is defined by the value of three channels, where the first represents the brightness, the second represents the color on a green to red scale and the third represents a color on a blue to yellow scale. $\mathrm{L}^{*} \mathrm{a}^{*} \mathrm{~b}^{*}$ color model gives the Munsell system of color classification [33].

\section{EXPERIMENTATION}

The retrieval tests are carried out in order to verify which color descriptor method gives good retrieval results based on highest recall and precision parameters. Performance of different color models is also evaluated. Further the best two methods have been evaluated using a single performance parameter i.e. Mean average precision(MAP) for all color models. To verify the applicability of color models and effective color feature extraction methods for semantically meaningful image retrieval, the results are noted for three semantic classes that are Manmade Indoor, Mandmade Outdoor and Natural Outdoor.

In these experiments, two databases are used. One is our own database created by collecting 450 images from 10 different categories i.e. store, bedroom, street, building, buses, coast, forests, snowy mountain, twilight and kitchen as shown in Figure 1(a). Another is the standard database of James Z. Wang et.al.[25] and it is a subset of 1000 images of the Corel database which were selected manually to form 10 classes of 100 images each. The images are subdivided into 10 sufficiently distinct classes (e.g. buildings, buses, African people, food plates, beaches, dinosaurs, elephants, roses, horses and snowy mountains as shown in Figure 1(b)) such that it can be assumed that a user wants to find the other images from the class if the query is from one of these classes. The database is created at Pennsylvania University and is publically available. 


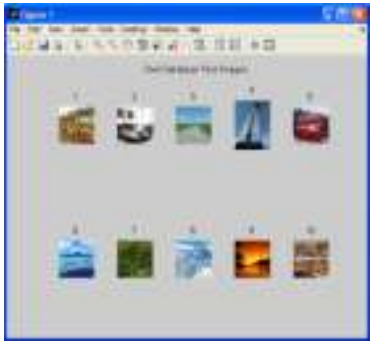

(a) (b)

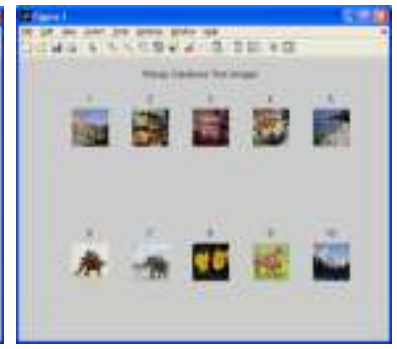

Fig 1: Test Images from (a) Own Database (b) Wang's Database

The experimentation involves two stages, training and testing for image retrieval. Training procedure is shown in Figures 2(a) and Figure 2(b). In the first stage, database images are converted into desired color models and their features are computed using selcted color descriptor method. Structures for four semantic sub-classes like manmade,natural,indoor and outdoor are formed by providing features matrix of database images and the targets corresp onding to these classes to the SVM for training. Linear Kernel as well as non linear kernel i.e. Gaussian Radial Basis Function(RBF) [34] is used and it is observed that RBF outperformed the Linear Kernel in both accuracy and convergence time. Therefore in this paper, results using RBF kernel are shown. Linear kernel is used only for color moments method.

Second stage consists of image retrieval procedure which is shown in Figure 3. Given a query image, the color model conversion is carried out, its color feature vectors are computed. By using these color features and the structures obtained for four sub-classes, SVM classifiers classify the

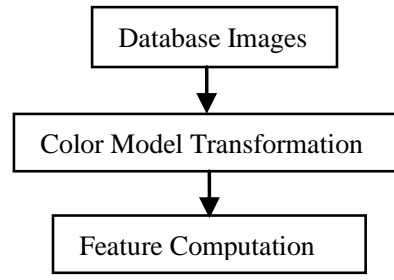

Fig 2(a)

query image into sub-classes. Further, the query image is identified as one of the three main classes that are Manmade indoor, Manmade Outdoor or Natural outdoor. In addition to this, the query image features are compared with those of the database images using Euclidean distance as it is the most commonly used distance metric for image retrieval. Experimentation is done using Quadratic distance but more semantically relevant images are retrieved using Euclidean distance.

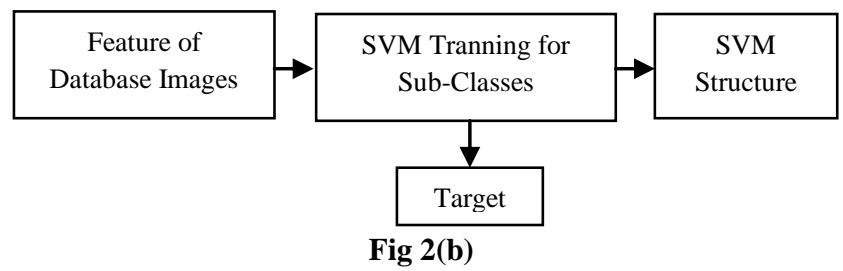

Fig 2: Training Process: (a) Feature Computation of database images and (b) Formation of SVM Structures

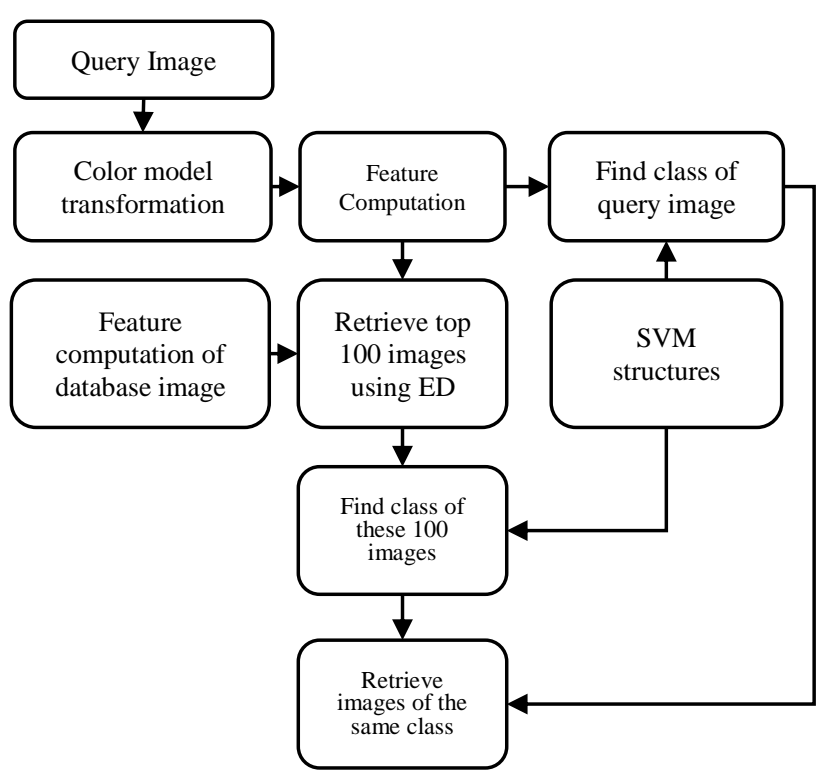

Fig 3: Image Retrieval Process

In this paper, results based on only Euclidean distance (ED) are presented. Top 100 images having minimum distance are retrieved. From these 100 images, only images having the same class as that of the query image are displayed.

The color descriptors given in the literature i.e. $\mathrm{CH}[27]$, $\mathrm{CM}$ [28] and CCV[30] are used here and their performance is compared with our proposed methods based on the combination of these descriptors i.e. $\mathrm{CH}-\mathrm{CM}$, $\mathrm{CH}-\mathrm{CCV}$, $\mathrm{CCV}-\mathrm{CM}, \mathrm{CH}-\mathrm{CM}-\mathrm{CCV}$. Also every method is implemented using different color models. Performance parameters used for the assessment of color models and color descriptors are Precision, Recall and Mean Average Precision (MAP). MAP term not only gives the retrieval efficiency but also shows how quickly the image is retrieved. Hence rank wise image retrieval can be evaluated. Performance Parameters [35-38] used for comparing various color models as well as various color descriptors are given by the equations (4) to (6).

$$
\begin{aligned}
& \text { Pr ecision }=\frac{\text { No. of relevant images retrieved }}{\text { Total No. of images retrieved }} \\
& \text { Precision }(r)=\frac{\text { No. of relevant images retrieved at rank } r}{r} \\
& \operatorname{Re} \text { call }=\frac{\text { No. of relevant images retrieved }}{\text { Total No. of relevant images in the database }}
\end{aligned}
$$

The average precision is defined as the mean precision after every relevant image is retrieved. The parameter MAP as shown in equation (7) is the mean of the precision for each relevant image is retrieved. MAP measures the average performance of multiple retrieved images.

$$
M A P=\frac{\sum_{r=1}^{N}(\operatorname{pre}(r) \times \operatorname{rel}(r))}{\text { No. of relevant images in the database }}
$$

Where $\mathrm{N}$ is the total No. of retrieved images, $\mathrm{r}$ is the rank of the retrieved image; rel(r) is a binary function $(0$ or 1$)$ indicating whether the retrieved image at the given rank $r$ is relevant to the query image, and pre(r) is the precision at the given rank $r$. This measure favors the features that can retrieve relevant images earlier. 


\section{RESULTS AND DISCUSSION}

Initially the experiments are done on our own database Sample query images are shown in Figure 1. Figure 4 shows the retrieval results for the query image 'Building' (similar to the image in topmost corner of Figure 4) when it is transformed into I1I2I3 color model. The feature extraction method used is CCV-CH-CM. Class of this query is identified as "Manmade Outdoor".

Precision-Recall graphs for all the color models are shown in Figure 5. It is observed that I1I2I3 model gives the best

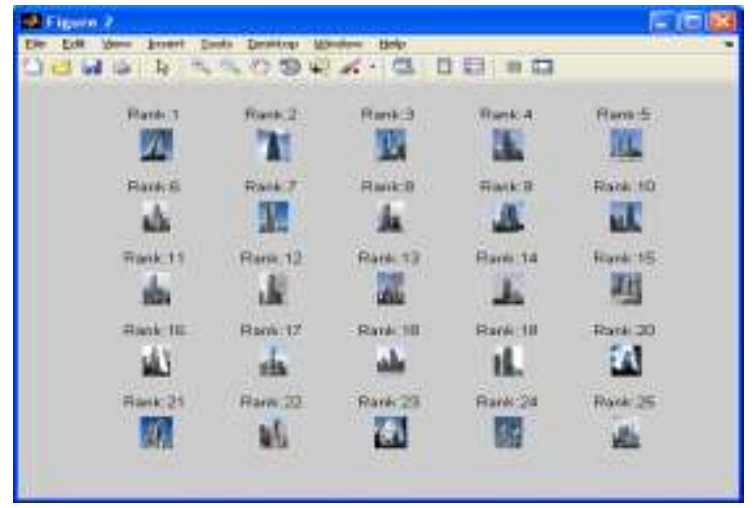

retrieval accuracy (Recall-0.84) when compared with other models.

The similar experiment is done on sample query images from each category with all color models and color feature extraction methods and results are obtained. Results for highest Recall and corresponding color models for all color feature extraction methods are shown in Table 1 and Table 2 respectively.

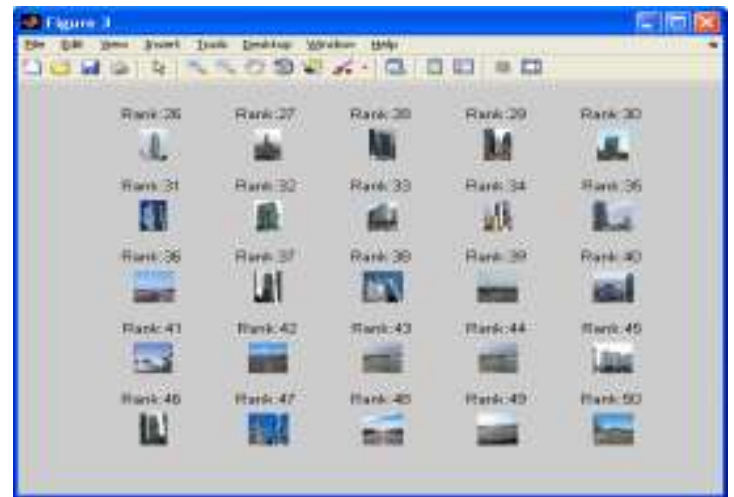

Fig 4: Retrieval Results for 'Building' Image (similar to the image at 'Rank 1') with I1I2I3 color model transformation using proposed method (CCV-CM-CH)

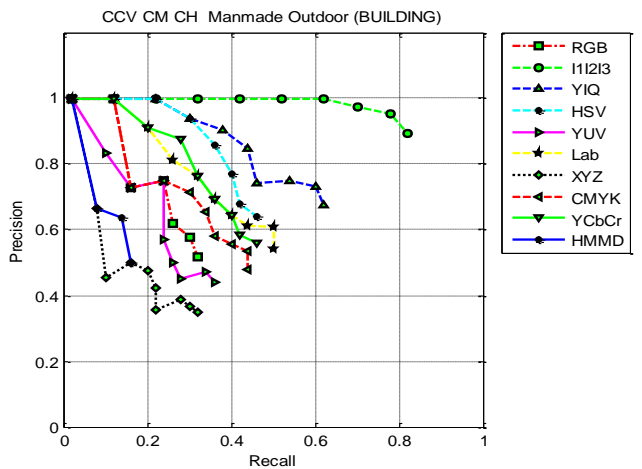

Fig 5: Precision-Recall Graphs for 'Building' image using different color models

Table 1. Maximum Recall Values Obtained by All color feature extraction methods on Own Database

\begin{tabular}{llllllll}
\hline Image Category & CH & CM & CCV & CH-CM & $\begin{array}{l}\text { CCV- } \\
\text { CM }\end{array}$ & $\begin{array}{l}\text { CCV- } \\
\text { CH }\end{array}$ & $\begin{array}{l}\text { CCV- } \\
\text { CM-CH }\end{array}$ \\
\hline Store & 0.84 & 0.54 & 0.76 & 0.72 & $\mathbf{0 . 9 4}$ & 0.7 & 0.72 \\
Bedroom & 0.72 & 0.48 & 0.54 & 0.6 & 0.68 & 0.72 & $\mathbf{0 . 8}$ \\
Street & 0.56 & 0.4 & 0.58 & 0.54 & 0.64 & $\mathbf{0 . 6 6}$ & 0.58 \\
Building & 0.74 & 0.52 & 0.84 & 0.86 & $\mathbf{0 . 9 6}$ & 0.84 & 0.84 \\
Bus & $\mathbf{1}$ & 0.72 & 0.84 & 0.92 & 0.94 & 0.98 & 0.96 \\
Coast & 0.64 & 0.5 & 0.5 & 0.7 & 0.64 & 0.72 & $\mathbf{0 . 7 6}$ \\
Forest & 0.94 & 0.96 & 0.94 & $\mathbf{0 . 9 8}$ & 0.96 & $\mathbf{0 . 9 8}$ & $\mathbf{0 . 9 8}$ \\
\hline Snow Mountain & $\mathbf{1}$ & 0.68 & 0.6 & $\mathbf{1}$ & $\mathbf{1}$ & 0.88 & 0.92 \\
\hline Twilight & $\mathbf{0 . 9 8}$ & 0.92 & 0.8 & $\mathbf{0 . 9 8}$ & 0.94 & $\mathbf{0 . 9 8}$ & $\mathbf{0 . 9 8}$ \\
\hline Kitchen & 0.52 & 0.48 & 0.76 & 0.44 & 0.8 & 0.64 & $\mathbf{0 . 8 4}$ \\
\hline
\end{tabular}


Table 2. Optimum Color Models for All Methods (Own Database)

\begin{tabular}{llllllll}
\hline Image Category & CH & CM & CCV & CH-CM & $\begin{array}{l}\text { CCV- } \\
\text { CM }\end{array}$ & CCV-CH & $\begin{array}{l}\text { CCV- } \\
\text { CM-CH }\end{array}$ \\
\hline Store & HMMD & L*a*b* & HMMD & RGB & HMMD & YUV,RGB & RGB \\
Bedroom & CMYK & YCbCr & HMMD & L*a*b* & YCbCr & YIQ & L*a*b* \\
Street & HMMD & L*a*b* & I1I2I3 & I1I2I3 & I1I2I3 & L*a*b* & I1I2I3 \\
Building & I1I2I3 & I1I2I3 & I1I2I3 & I1I2I3 & L*a*b* & I1I2I3 & I1I2I3 \\
Bus & HMMD & L*a*b* & YCbCr & HSV & YCbCr & HSV & HSV \\
Coast & YCbCr & YCbCr & HSV & L*a*b* & HMMD & CMYK & L*a*b* \\
Forest & YCbCr & CMYK & I1I2I3 & L*a*b* & I1I2I3 & HSI & L*a*b* \\
Snow Mountain & YCbCr & CMYK & HSV & YCbCr & CMYK & YIQ & HMMD \\
Twilight & YIQ & HMMD & YUV & I1I2I3 & YUV & YIQ & YIQ \\
Kitchen & YIQ & RGB & YIQ & L*a*b* & CMYK & L*a*b* & L*a*b* \\
\hline
\end{tabular}

It is marked that the results are improved by our proposed methods based on the combination of two or all color descriptors. So, for further analysis of color models, the best performed two methods that are CCV-CM and CCV-CM-CH are considered. Here, the same experiments are done on
Wang's standard database. The retrieval results for one of the 'Bus' images are shown in Figure 6. Precision-Recall plots by $\mathrm{CCV}-\mathrm{CM}-\mathrm{CH}$ method for all color models are shown in Figure 7. It is observed that I1I2I3 color spaces gives good retrieval accuracy.

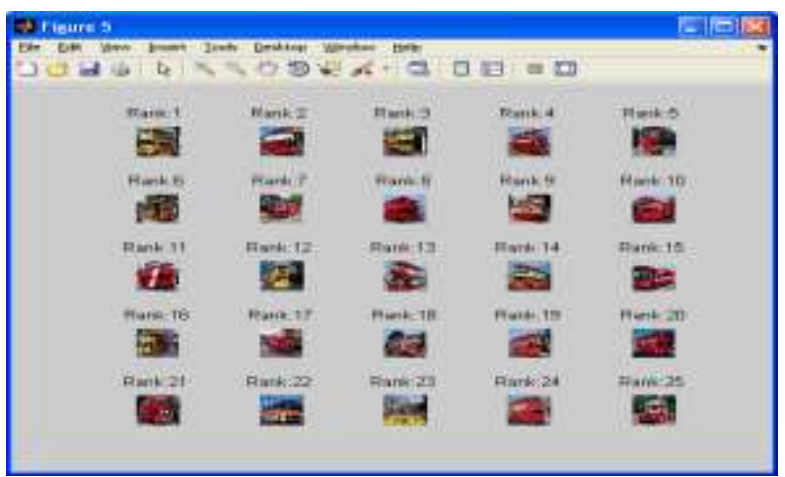

(a)

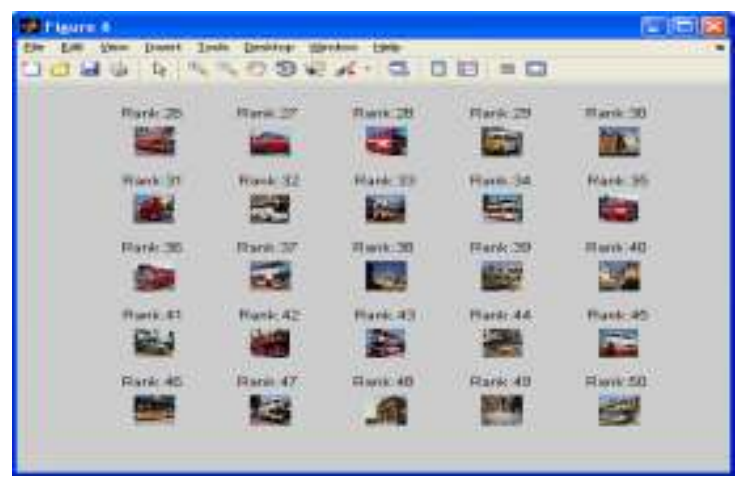

(b)

Fig 6 (a) and (b): Retrieval Results for 'Bus' Image with I1I2I3 color model transformation using proposed method (CCV-CMCH)

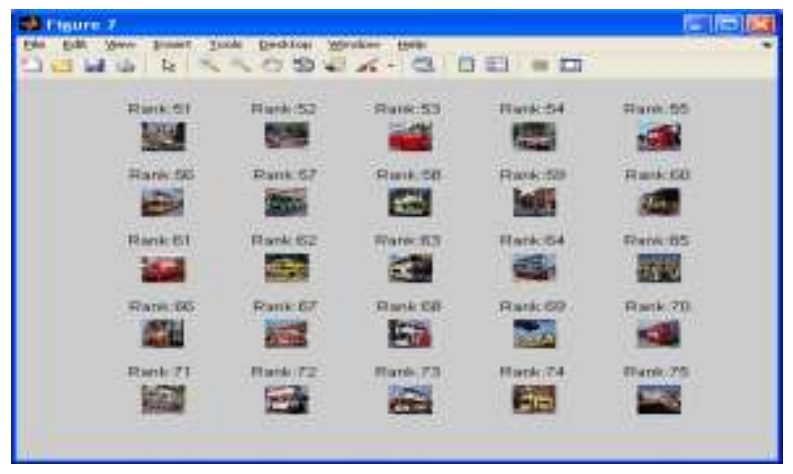

(c)

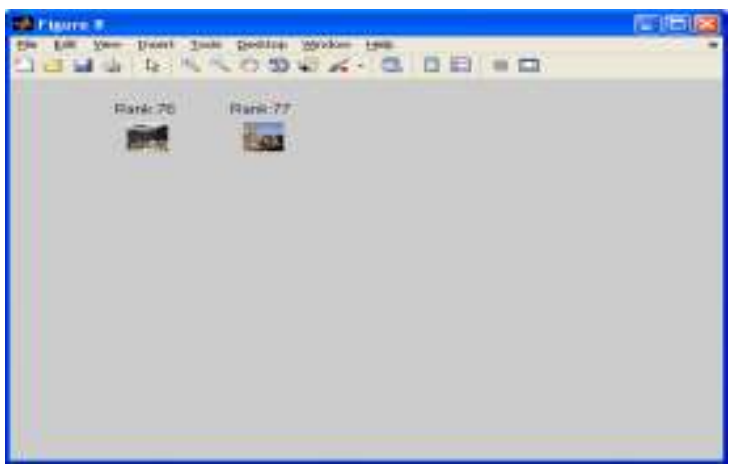

(d)

Fig 6 (c) and (d): Retrieval Results for 'Bus' Image with I1I2I3 color model transformation using proposed method (CCV-CMCH) 

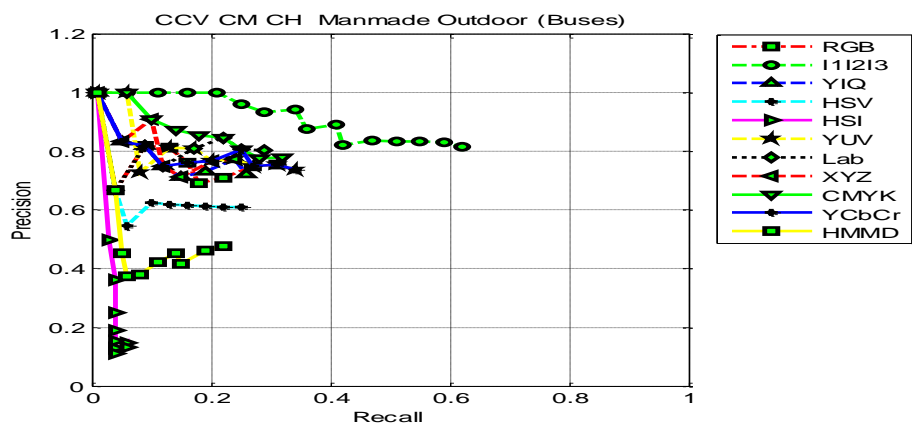

Fig 7: Precision-Recall Graph for 'Bus' image using different color models

In CBIR, rank-wise retrieval of semantically meaningful images is of prime importance. To find out how quickly the semantically meaningful images are retrieved, MAP parameter which is based on the retrieval rank of positive images is calculated. This is obvious from Table(3). Though precision is same for HSV and XYZ transformations, and recall is same for all the three transformations, the MAP values are different. In case of $\operatorname{HSV}(\operatorname{MAP}(0.3))$, more relevant images are retrieved at earlier rank than other two cases(MAP (0.27) and (0.28)).

Table 3. Retrieval Results for 'Building' Category from Wang's Database Using CCV-CM-CH Method

\begin{tabular}{lccc}
\hline Parameters & RGB & HSV & XYZ \\
\hline No. of relevant images retrieved & 33 & 33 & 33 \\
Total No. of images retrieved & 47 & 44 & 44 \\
No. of relevant images in the database & 100 & 100 & 100 \\
Precision & 0.70 & 0.75 & 0.75 \\
Recall & 0.33 & 0.33 & 0.33 \\
MAP & 0.27 & 0.3 & 0.28 \\
\hline
\end{tabular}

To optimize the semantic gap in CBIR, it is required to find the suitable color model for particular semantic class of images. Therefore an average MAP for different semantic classes like Manmade indoor, Manmade outdoor and Natural outdoor using all color models is computed as shown in Figure 8. Horizontal axes show the color models from 1 to 10 in the order of RGB,I1I2I3, YIQ, HSV,YUV, L"a* ${ }^{*}$, XYZ, CMYk, YCbCr and HMMD respectively.

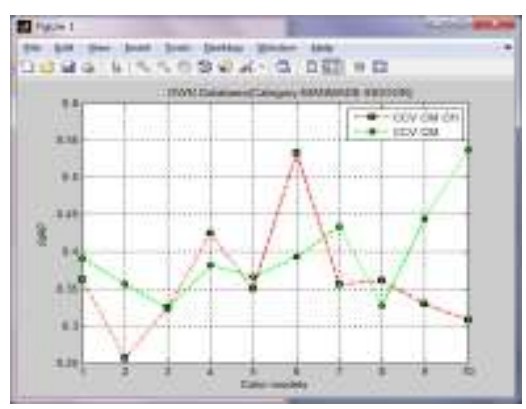

(a)

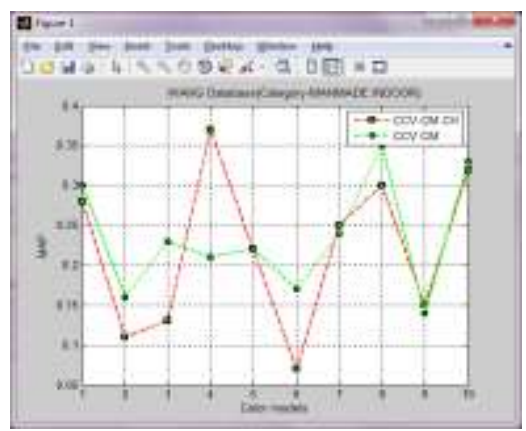

(d)

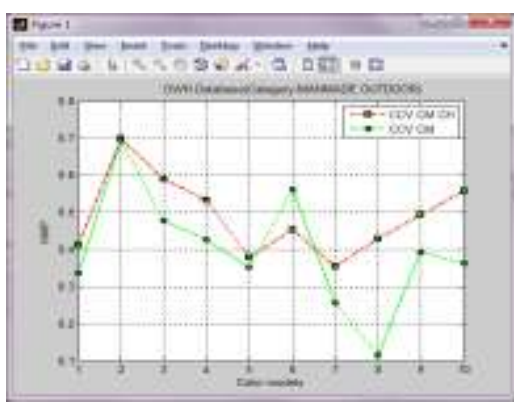

(b)

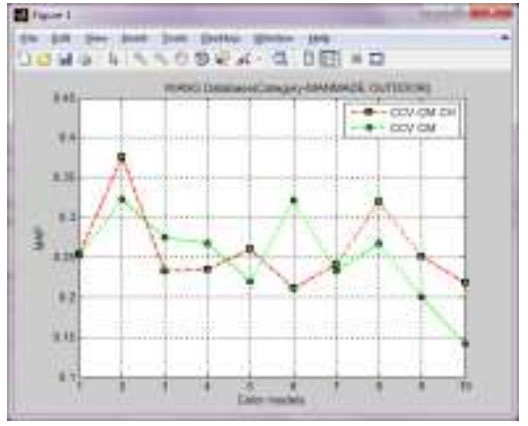

(e)

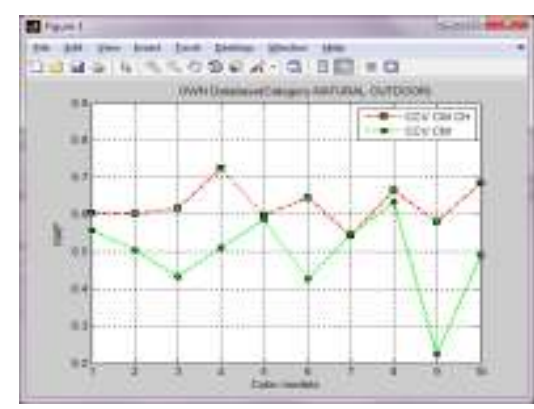

(c)

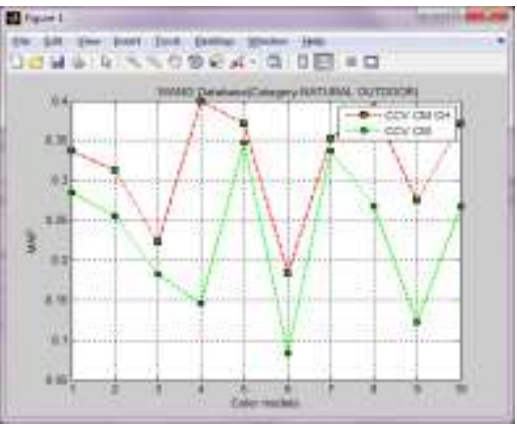

(f)

Fig 8 : Graphs for Average-MAP Vs Color Models for our 'Own' synthesized Database ((a),(b),(c)) and Wang's Standard Database ((d),(e),(f)) for three semantic classes such as Manmade Indoor ,Manmade Outdoor and Natural Outdoor 
From own database, images of store, bedroom and kitchen categories are considered as Manmade indoor; Street, buildings and bus images are considered as Manmade outdoor whereas coast, forest, snow mountains and twilight images are from Natural outdoor category. Similarly from Wang's database, food images are considered as Manmade indoor, buildings and bus categories as Manmade outdoor and Natural outdoor class consists of images of Africans, beach, dinosaurs, elephants, roses, horses and snowy mountains.

It is marked that the more improved retrieval results are obtained by using our proposed method i.e. CCV-CM-CH for feature extraction. The primary color model RGB shows comparatively better results only for natural images as compared to manmade images. The HSV color model proves to be the best for Natural outdoor images as it describes the perceptual color relationship more accurately than RGB and other color models. Another perceptually uniform color model HMMD and secondary color model CMYk are also found to give good rank-wise retrieval results for Natural outdoor images. However, HSV may be the best choice for Natural outdoor class as the computational time required for HMMD and CMYk is very high as compared to HSV. Though non correlated color model I1I2I3 is usually preferred for natural images [31], from our results it is marked that this model gives best semantically meaningful results for Manmade outdoor class of images. It can be inferred from the results on our own database that perceptual color models such as L*a*b* , HSV and HMMD performed well in Manmade indoor class of images but experiments on Wang's database do not confirm the efficiency for $\mathrm{L}^{*} \mathrm{a} * \mathrm{~b} *$ model.

\section{CONCLUSION AND FUTURE SCOPE}

In this work, the performance of a wide variety of color models in combination with different color descriptors for semantically meaningful content based image retrieval is presented and evaluated. The main question addressed in this paper is that which color model is suitable along with which color descriptors so that it will be helpful to optimize the semantic gap. In the current work, performance parameters i.e. Precision, Recall and MAP (mean Average Precision) are used. Our experimental results suggest that the proposed approach gives a good evaluation of the applicability of color models for the reduction of semantic gap. Different color models along with different color descriptors mentioned in the proposed methods can be applied for the image retrieval of more semantically meaningful images. It should be noted that the proposed methodology may be further improved by using more classes so as to get more semantically relevant results. By combining other features such as texture, shape, etc. along with color can improve retrieval results close to human interpretation. To reduce semantic gap further for large databases, one may use database reduction techniques like hue histogram reduction method [39].

\section{REFERENCES}

[1] Ying Liu,Dengsheng Zhang,Guojun Lu and Wie- Ying Ma,"A survey of content-based image retrieval with high-level semantics", Pattern Recognition,vol.40, issue1, pp262-282, January 2007.

[2] M.B .Kokare, B.N. Chatterji and P.K. Biswas, "A Survey On Current Content Based Image Retrieval Methods", IETE Journal of Research, 2002,pp. 261-271.

[3] G.Rafiee, S.S.Dlay, and W.L.Woo, "A Review of ContentBased Image Retrieval”, CSSNDSP 2010,pp.775-779.
[4] Thomas Sikora, "The MPEG-7 Visual Standard for Content Description-An Overview", IEEE Transactions on Circuits and Systems for Video Technology, vol. 11, No. 6, June 2001, pp.696-702

[5] Wei Bian and Dacheng Tao, Member, IEEE, "Biased Discriminant Euclidean Embedding for CBIR", IEEE Transaction on Image processing, vol. 19, no. 2, pp.545554, February 2010.

[6] Pranoti Mane and Dr. N.G.Bawane, "Optimization of gap between Visual Features and high level Human Semantics in Content Based Image Retrieval", SCITECH-2012 ,S.B.Patil College of Engineering, Pune, January 2012.

[7] Pranoti Mane and Dr. N.G.Bawane , "Comparative Performance Evaluation of Edge Histogram Descriptors and Color Structure Descriptors in Content Based Image Retrieval", IJCA Proceedings on NCIPET 2013, No.6, pp. 5-9, December 2013.

[8] Agma J. M. Traina, Joselene Marques, Caetano Traina Jr, "Fighting the Semantic Gap on CBIR Systems through New Relevance Feedback Techniques", Proceedings of the 19th IEEE Symposium on Computer-Based Medical Systems (CBMS'06)

[9] Arnold W. M. Smeulders, Marcel Worring, Simone Santini, Amarnath Gupta, and Ramesh Jain, "Contentbased image retrieval at the end of the early years.", IEEE Trans. Pattern Anal. Mach. Intell., 22(12),pp.13491380,2000

[10] O.Karam, A.Hamad, and M.Attia,"Exploring the Semantic Gap in CBIR: with application to Lung CT", GVIP 05 Conference, CICC, Cairo,pp. Egypt,pp.422426, 19-21 December 2005.

[11] J. Tao, X. Tang, X. Li, and X. Wu, "Asymmetric bagging and random subspace for support vector machines-based relevance feedback in image retrieval," Pattern Analysis and Machine Intelligence, IEEE, vol. 28, pp.1088- 1099, 2006.

[12] R. Gonzalez and R.Woods, "Digital Image Processing. Reading", Third Edition, Pearson Education, ISBN 97881-317-2695-2, 2009

[13] Sung Ha Kang and Riccardo March, "Variational Models for Image Colorization via Chromaticity and Brightness Decomposition", IEEE Trans. Image Process., vol.16, no. 9, pp. 2251-2261, September 2007.

[14] P. Blomgren and T. F. Chan, "Color TV: Total variation methods for restoration of vector-valued images," IEEE Trans. Image Process., vol.7, no. 3, pp. 304-309, March 1998.

[15] T. F. Chan, S. H. Kang, and J. Shen, "Total variation denoising and enhancement of color images based on the CB and HSV color models," J. Vis. Comm. Image Represent., vol. 12, no. 4, pp. 422-435, 2001.

[16] P. Perona, "Orientation diffusion," IEEE Trans. Image Process., vol. 7,no. 3, pp. 457-467, March 1998.

[17] G. Sapiro and D. Ringach, "Anisotropic diffusion of multivalued images with applications to color filtering," IEEE Trans. Image Process.,vol. 5, pp. 1582-1586, May 1996. 
[18] B. Tang, G. Sapiro, and V. Caselles, "Color image enhancement via chromaticity diffusion," IEEE Trans. Image Process., vol. 10, no. 5,pp. 701-707, May 2001.

[19] P. E. Trahanias, D. Karako, and A. N. Venetsanopoulos, "Directional processing of color images: Theory and experimental results," IEEE Trans. Image Process., vol. 5, no. 6, pp. 868-880, June 1996.

[20] W. Y. Ma and H. J. Zhang, "Benchmarking of image features for content-based image retrieval," in Proc. 32nd Asilomar Conf. Signals, Systems, Computers, Pacific Grove, CA, vol. 1, pp. 253-257, November 1998.

[21] Mircea C. Ionita, Peter Corcoran, and Vasile Buzuloiu, "On Color Texture Normalization for Active Appearance Models", IEEE Transactions on Image processing,Vol.18,No.6,pp.1372-1378, June 2009.

[22] M. B. Stegmann and R. Larsen, "Multi-band modeling of appearance," Image Vis. Comput., vol. 21, no. 1, pp. 6167, January 2003

[23] R. Neelamani, Ricardo de Queiroz, Zhigang Fan,Sanjeeb Dash, and Richard G. Baraniuk, "JPEG Compression History Estimation for Color Images", IEEE Transactions on Image Processing, vol. 1, no. 6, June 2006

[24] L. Zhang, F. Liu, B. Zhang, "Support vector machine learning for image retrieval", International Conference on Image Processing, pp. 7-10, October 2001.

[25] James Z. Wang, Database, http://wang.ist.psu.edu

[26] Robert Magnusson, Gustav Bladh, "Semantic Scene Classification for Enhanced Image Browsing Experience", Master Thesis, Lund University, April 2011

[27] F. Long, H.J. Zhang, D.D. Feng, "Fundamentals of content-based image retrieval", Multimedia Information Retrieval and Management, Springer, Berlin, 2003

[28] M. Flickner, H. Sawhney, W. Niblack, J. Ashley, Q. Huang, B. Dom, M. Gorkani, J. Hafner, D. Lee,D. Petkovic, D. Steele, and P. Yanker, "Query by image and video content: The QBIC system." IEEE Computer, vol.28, no.9, pp. 23-32, September 1995
[29] W. Niblack et al., "Querying images by content, using color, texture and shape", SPIE Conference on Storage and Retrieval for Image and Video Database, vol. 1908, pp.173-187, April 1993.

[30] Ramin Zabih Justin Miller, Greg Pass, "Comparing images using color coherence vectors", Conference,MM96, The fourth ACM International Multimedia Conference, Boston, MA, USA, pp. 65-73, November 18-22,1996.

[31] Y. I. Ohta, T. Kanade, and T. Sakai, "Color information for region segmentation", Computer Graphics and Image Processing, vol. 13, pp. 222-241, 1980.

[32] J.R. Ohmer, H. J. Kim, S. Krishnamachari ,B. S. Manjunath , Akio Yamada , "The MPEG-7 Color Descriptors".

[33] Petteri Kerminen and Moncef Gabbouj, "Image Retrieval Based on Color Matching", In Proceedings of the Finnish Signal Processing Symposium (FINSIG-99),1999.

[34] Asa-Ben Hur, Jason Weston, “A User's Guide to Support Vector Machines"

[35] Daan He, "Three new methods for color and texture based Image matching in content-based image retrieval", $\mathrm{PhD}$ Thesis, Dalhousie University Halifax, Nova Scotia, April 2010

[36] Vasileios Mezaris, Ioannis Kompatsiaris and Michael G. Strintzis, "An Ontology based approach to object based Image retrieval", ICIP 2003

[37] Samuel Barretta, Ran Changb and Xiaojun Qib, "Fuzzy based learning approach to CBIR", ICME2009, IEEE, pp 838-841, 2009

[38] Pierre Blanchart and Mihai Datcu, "A Semi-Supervised Algorithm for Auto-Annotation and unknown Structures Discovery in Satellite Image Databases", IEEE journal of selected topics in applied earth observations and remote sensing, vol. 3, no. 4,pp698-717, December 2010

[39] Nishant Singh, S. Dubey, P. Dixit, J.P. Gupta, "Semantic image retrieval by combining color,texture and shape features", IEEE International conference on computing Sciences, IEEE ICCS,pp.116-120, 2012. 